\title{
Inflammatory Response in Acute Lung Injury: A Comparison between Intratracheal and Intubation Administration of $P$.
}

\section{Aeruginosa Lipopolysaccharide}

\section{Di Gioia S*, di Toma L, Castellani S and Conese M \\ Department of Medical and Surgical Sciences, University of Foggia, Italy}

*Corresponding author: Di Gioia S, Department of Medical and Surgical Sciences, University of Foggia, Foggia, Italy, pin code: 71122, Tel: +390881588074; Fax: +390881588037; Email: sante.digioia@unifg.it

\section{Short Communication}

Volume 3 Issue 1

Received Date: February 04, 2018

Published Date: February 13, 2018
Abbreviations: ALI: Acute Lung Injury; BALF: Bronchoalveolar Lavage Fluid; CF: Cystic Fibrosis; LPS: Lipopolysaccharide; TR: Tracheotomy; IT: Intubation

\section{Introduction}

In humans, the hallmark of the acute lung injury (ALI) is a neutrophilic inflammatory response with fibrin-rich proteinaceous exudates containing an array of pro- and anti-inflammatory cytokines [1-3]. Among the animal model recapitulating these features, the administration of bacterial endotoxin (lipopolysaccharide, LPS), one of the major determinant of toxicity given by Gram negative bacteria, is extensively used to model ALI [4]. In general, ALI caused by LPS administration is characterized by neutrophil accumulation in the airspaces and elevation of cytokines and chemokines in the epithelial lining fluid.

Robust experimental ALI models are a prerequisite for understanding of the inflammatory response and the host defence mechanism against tissue injury. However, the route of administration is often associated with technical difficulties, especially where small experimental animals such as mice are concerned. These problems are relevant also for preclinical tests of strategies that aim to dampen the inflammatory response in ALI.

Pseudomonas aeruginosa is an opportunistic pathogen that causes urinary tract infections, respiratory system infections, dermatitis, and soft tissue infections. It also causes a variety of systemic infections, particularly in patients with severe burns and in cancer and AIDS patients who are immunosuppressed. Moreover, $P$. aeruginosa is a leading cause of hospital acquired pneumonia and of chronic airway infection in individuals with cystic fibrosis (CF) [5,6]. LPS from P. aeruginosa has been used to induce ALI in mice by intratracheal $[7,8]$ and intranasal injection [9].

Surprisingly, the P. aeruginosa LPS model of ALI in mice has not yet been comprehensively and systematically described. The present study aims to characterize the pulmonary inflammatory events produced by the tracheotomy of $P$. aeruginosa LPS in comparison with those determined by LPS intubation. This will facilitate ALI research and will promote our understanding of the pathogenesis of this complex disease.

\section{Methods}

The tracheotomy (TR) was carried out after anesthetizing C57Bl/6 mice intraperitoneally with 2,2,2tribromoethanol (Avertin, prepared 2.5\% weight/volume in PBS and used $0.015 \mathrm{ml} / \mathrm{g}$ body weight; Sigma) and fixing them on a board at an angle of $45^{\circ}$ in a supine position. After sterilization, a mid-line incision was performed in the neck to isolate the trachea. LPS $(20 \mu \mathrm{g})$ was diluted in $50 \mu \mathrm{l}$ sterile saline and intratracheally instilled with a 22 gauge-needle syringe as previously described [10]. Control mice received $50 \mu \mathrm{l}$ sterile saline. After intratracheal instillation, all mice were put in a vertical position to ensure that the fluid was evenly distributed in both lungs.

The intubation (IT) was performed as follows: Mice were anesthetized as described above and restrained in a 


\section{Open Access Journal of Pulmonary \& Respiratory Sciences}

vertical position with their necks tilted back so as to result in an accessible position of the trachea for intubation. Using two pairs of tweezers, the mouth was carefully opened and the tongue was gently pulled out. Then, each animal received a single $50 \mu$ bolus of LPS in saline $(20 \mu \mathrm{g})$ or $50 \mu \mathrm{l}$ of saline (control mice) introduced into the trachea using a 22-gauge intravascular cannula sleeve with the needle removed. To avoid false intubation into the esophagus, the trachea was illuminated via an otoscope held perpendicularly to the animal's throat, producing a well illuminated trachea through dispersed light.

In both protocols, to obtain bronchoalveolar lavage fluid (BALF) specimens, the trachea was exposed through a midline incision and cannulated with a sterile 23-gauge needle. BALF was performed by instilling $0.9 \% \mathrm{NaCl}$ in two separate 1-ml aliquots. BALF was performed to determine the total cell numbers using a hemocytometer. Smears of BALF cells were stained with Giemsa to determine the neutrophil counts. The concentrations of MIP-2 in the BALF were measured with enzyme immunoassays, according to the manufacturer's protocols.

\section{Results and Conclusions}

In this study we were interested in evaluating which method (between IT and TR) is the most efficient to deliver Ps. aeruginosa LPS directly to the lungs of mice so as to modelling an ALI. More specifically, if we directly compare the effects of administration of the same dose of LPS by the more invasive TR as compared to IT route, do we see the same effects qualitatively and quantitatively? First, we assessed the inflammatory response in the lung of mice by evaluating the cellular presence and phenotype in the BALF at various time points $(24,48$ and 72 hours) following the LPS administration. Fig. 1A demonstrates that the endotoxin instillation induced a significant increase in the total cell count of BALF, by either routes of administration, as compared to mice injected with saline solution. To further examine the basis for these changes, we examined the cell differentials in the BALF from both groups of mice. The instillation of LPS is usually associated with an acute neutrophilic influx, thus we hypothesized that even in our experiments, the massive increase of total cell counts in the BALF was attributable to neutrophil infiltration. As shown in Fig. $1 B$, in the cytocentrifuge fields evaluated we observed that the BALF from mice treated with LPS by IT, approximately $84 \%$ of cells were neutrophils. Interestingly, this neutrophilia was maintained at each time point tested, whereas mice treated with LPS by TR showed a less pronounced neutrophilia, with the neutrophils percentage increasing from about $50 \%$, at $24 \mathrm{~h}$, up to about $70 \%$ in the following 48-72 h. No statistically significant differences were found between LPS-treated TR vs. IT mice (Kruskal-Wallis oneway analysis of variance). Next, BALF was evaluated to assess the levels of one of the most important CXC chemokine involved in neutrophils recruitment: MIP-2 (macrophage-inflammatory protein-2). As shown in Fig. 1C, MIP-2 levels both in the LPS-IT mice and LPS-TR mice peaked at the 48-h time and, interestingly, at each time point following LPS-stimulation, MIP-2 protein levels of IT mice were greater than TR ones. However, no statistically significant differences were found between LPS-treated TR vs. IT mice.

In conclusion, LPS instilled in lung mice can mimic the early phases of ALI pathogenesis occurring in humans. However, our results are entirely consistent with the intubation route being equivalent to tracheotomy in terms of induction of an acute inflammatory response in mouse lungs. Therefore, intubation should be preferred to tracheotomy, which requires invasive surgery and is accompanied by pain and discomfort to the animals.
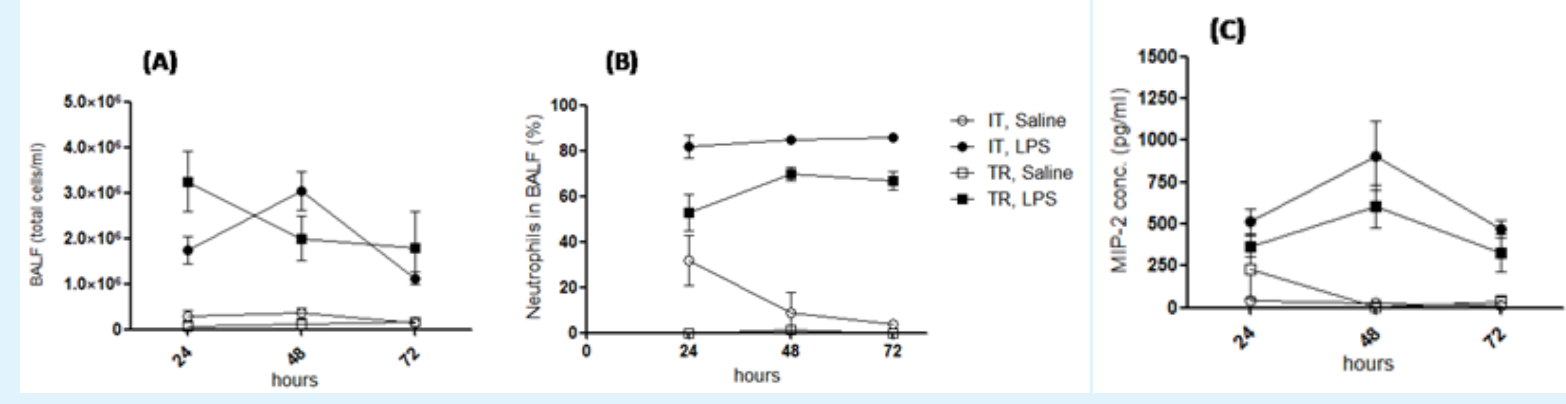

Figure 1: Effect of route administration (IT, intubation versus TR, tracheotomy). (A) Bronchoalveolar fluid (BALF) total cell counts; (B) BALF neutrophil percentages; (C) BALF MIP-2 concentration. Each value is the mean \pm SEM (error bar). $\mathrm{n}=3-6$ mice 


\section{Open Access Journal of Pulmonary \& Respiratory Sciences}

\section{References}

1. Pittet JF, Mackersie RC, Martin TR, Matthay MA (1997) Biological markers of acute lung injury: prognostic and pathogenetic significance. Am J Respir Crit Care Med 155(4): 1187-1205.

2. Butt Y, Kurdowska A, Allen TC (2016) Acute Lung Injury: A Clinical and Molecular Review. Arch Pathol Lab Med 140(4): 345-350.

3. Tsushima K, King LS, Aggarwal NR, De Gorordo A, D'Alessio FR, et al. (2009) Acute lung injury review. Intern Med 48(9): 621-630.

4. Matute-Bello G, Frevert CW, Martin TR (2008) Animal models of acute lung injury. Am J Physiol Lung Cell Mol Physiol 295(3): 379-399.

5. Arancibia F, Bauer TT, Ewig S, Mensa J, Gonzalez J, et al. (2002) Community-acquired pneumonia due to gram-negative bacteria and pseudomonas aeruginosa: incidence, risk, and prognosis. Arch Intern Med 162(16): 1849-1858.

6. Gibson RL, Burns JL, Ramsey BW (2003) Pathophysiology and management of pulmonary infections in cystic fibrosis. Am J Respir Crit Care Med 168(8): 918-951.

7. Yanagihara K, Seki M, Cheng PW (2001) Lipopolysaccharide Induces Mucus Cell Metaplasia in Mouse Lung. Am J Respir Cell Mol Biol 24(1): 66-73.

8. Hirakata $\mathrm{Y}$, Kirikae $\mathrm{T}$, Kirikae $\mathrm{F}$, Yamaguchi $\mathrm{T}$, Izumikawa K, et al. (1999) Effect of Pseudomonas aeruginosa exotoxin $\mathrm{A}$ on endotoxin-induced tumour necrosis factor production in murine lung. J Med Microbiol 48(5): 471-477.

9. Wieland CW, Siegmund B, Senaldi G, Vasil ML, Dinarello CA, et al. (2002) Pulmonary inflammation induced by Pseudomonas aeruginosa lipopolysaccharide, phospholipase $\mathrm{C}$, and exotoxin $\mathrm{A}$ : role of interferon regulatory factor 1 . Infect Immun 70(3): 1352-1358.

10. Trotta T, Di Gioia S, Piro D, Lepore S, Cantatore $S$, et al. (2013) Effect of acute lung injury on VLA-4 and CXCR4 expression in resident and circulating hematopoietic stem/progenitor cells. Respiration 85(3): 252-264. 